\title{
Entwicklung einer Fehleraufsuchdidaktik und Erwägungsorientierung - unter Berücksichtigung von Beispielen aus dem Grundschulunterricht
}

\section{Bettina Blanck}

In dem Beitrag werden zunächst Konzepte für einen positiven, d. h. konstruktiven Umgang mit Fehlern in Lern- und Lehrprozessen vorgestellt und gegenüber fehlervermeidenden Konzepten abgegrenzt. Danach wird auf die Unterscheidung zwischen "guten" und "schlechten Fehlern" eingegangen. Dabei wird deutlich, dass das, was als Fehler bezeichnet wird, sehr heterogen sein kann. Beispiele für Fehler aus dem Alltag von Grundschulunterricht belegen diese Einschätzung. Aus erwägungsdidaktischer Sicht ist es deshalb in zweifacher Hinsicht sinnvoll, eine "Fehleraufsuchdidaktik» zu entwickeln. Diese sollte darin unterstützen, «echte Fehler» zu identifizieren und "reflexive Fehlerkompetenzen" zu entwickeln. Ausserdem sollte eine "Fehleraufsuchdidaktik» für einen konstruktiven Umgang mit Fehlern die "Erwägungs-Geltungsbedingung» nutzen.

Vorbemerkung zum Stand der Entwicklung des Ansatzes

Ausgang der folgenden Überlegungen ist das Konzept eines erwägungsorientierten Umgangs mit Vielfalt und insbesondere mit Alternativen. Nach diesem Konzept sind die zu einem Auswahlgedanken (z. B. Frage, Problem) jeweils problemadäquat zu erwägenden Alternativen - selbst wenn sie als Lösungen falsch, ungünstig oder unsinnig wären - zentral für die Begründung der (vorerst) richtigen Lösung. Für Lösungsbegründungen sind die erwogenen Alternativen als eine Geltungsbedingung zu bewahren. Praktische Erfahrungen in Forschung, Lehre und Grundschulunterricht haben deutlich werden lassen, dass das Erwägungskonzept damit auch einen spezifischen Umgang mit Fehlern in Lern- und Lehrzusammenhängen impliziert. Hierfür sind einerseits theoretisch-konzeptuelle Klärungen zu leisten, andererseits sind Wege zur Veränderung der Lehrund Lernpraxis aufzuzeigen, welche dann auch zu evaluieren und zur konzeptuellen Verbesserung und Weiterentwicklung zu nutzen wären. Auf dem Weg zu einer solchen Rückkopplung aus theoretisch-konzeptueller Arbeit und Erfah- 
rungen in konkreten Lern- und Lehrzusammenhängen ist der vorliegende Beitrag zu verorten:

Es lässt sich anhand unterschiedlicher Verwendungsweisen des Terminus «Fehler» zeigen, wie heterogen der Ausdruck z. B. im Grundschulunterricht gebraucht wird. Die Vielfalt der Beispiele ist auch als Ausgangspunkt für eine differenziertere, reflexive Verwendung des Terminus zu nutzen. Eine erwägungsorientierte Begriffsklärung strebt eine beispielsbezogene Verortung des jeweiligen Begriffs in Bezug auf Ober- und Unterbegriffe mit ihren alternativen Konkretionen an. Reflexiv ist sie gegenüber alternativen Begriffsklärungen zu bedenken, wofür man allerdings zunächst einmal einen Klärungsvorschlag benötigt. Hierfür werden erste Vorschläge gemacht. Die Perspektive des Ansatzes geht dahin, eine konzeptuelle Grundlage für die Aus- und Fortbildung von Lehrerinnen und Lehrern und ihren Umgang mit Fehlern in Schule und Unterricht zu schaffen.

Insofern sich eine bloss negative Bewertung von Fehlern hin zu einem konstruktiven Umgang wandelt, bei dem Fehler sogar als «Helfer» oder für «negatives Wissen», das einen Beitrag zum "positiven Wissen» leistet, geschätzt werden, gibt es hier für einen erwägungsorientierten Ansatz wichtige Anknüpfungspunkte. Der Artikel beginnt deshalb mit einer kurzen Beschreibung des Forschungsstandes.

\section{Unterschiedliche Konzepte für den Umgang mit Fehlern in Lern- und Lehrprozessen}

Nach Weinert reicht das Spektrum möglicher Umgangsweisen mit Fehlern in Lern- und Lehrprozessen «vom postmodernistischen, kreativitätssüchtigen Fehlerkult» bis zur "traditionalistischen Fehlerverteufelung» (1999, S. 101). Ein wesentlicher Grund für unterschiedliche Umgangsweisen mit Fehlern ist, ob man eher die Auffassung eines vornehmlich vorgabeorientierten Lehrens und Lernens, einer "Belehrungskultur», oder aber eines eher entscheidungsorientierten Lernens und Lehrens vertritt. Insofern Belehrung häufig mit Abfragen einhergeht, bei dem geprüft und sanktioniert wird, was die Lernenden gelernt bzw. nicht gelernt haben, werden Fehler als Mängel aufgefasst, die die Lehrenden aufspüren müssen und die sowohl Lehrende wie Lernende zu vermeiden trachten. Es wird befürchtet - so etwa von Heuer für den Englischunterricht -, dass ein «einmal gemachter Fehler ... durch seine Korrektur nicht aufgehoben» wird: «Ein einmal gemachter Fehler lebt weiter. Auch die sofortige Korrektur durch den Lehrer kann seine Wirkung nicht ganz rückgängig machen. Es kommt also darauf an, von vornherein zu verhindern, dass die Schüler/innen überhaupt Fehler begehen» (1968, S. 64). ${ }^{1}$ Eine Konsequenz der «Strategie der Vermeidung, Unterdrückung, Sanktionierung, schnellstmöglichen Ausmerzung bzw. Korrektur von Fehlern (Fehler im Keim ersticken)", bei einer "Defiziteinstellung" gegenüber Fehlern, wird dabei häufig ein kleinschrittiges Vorgehen der Lehren- 
den sein (Wiater, 2004, S. 7). Wenn hier «aus Fehlern gelernt» wird, so ist dies wohl zumeist mit «negativen» Gefühlen verbunden. Denn Fehler «bieten Anlass für Schimpfen, Zur-Schau-Stellen, Häme, Blossstellung, Verlachtwerden, Schuldzuweisungen, Unterstellungen, Bestrafungen, Enttäuschtsein, Liebesentzug, Selbstwertverlust, Scham, Ärger, Wut ...» (a. a. O., S. 4). Wohin eine solche Lernatmosphäre führen kann, hat z. B. Dewey beschrieben:

Insistence upon avoiding error instead of attaining power tends also to interruption of continuous discourse and thought. Children who begin with something to say and with intellectual eagerness to say it are sometimes made so conscious of minor errors in substance and form that the energy that should go into constructive thinking is diverted into anxiety not to make mistakes, and even, in extreme cases, into passive quiescence as the best method of minimizing error. (1978, S. 326). ${ }^{2}$

Solches gilt nicht nur für Lern- und Lehrzusammenhänge, sondern auch in anderen Lebensbereichen, etwa der Berufswelt. Kahl moniert diesbezüglich: «Die Suche nach Fehlern schafft die Atmosphäre braver Planerfüllung, aber sie schafft kein Klima, das dazu stimuliert, Neues zu wagen» (1995, S. 21). Wer in diesem Sinne befürchtet, dass eine Konzentration auf Fehlervermeidung zur Einschränkung von Lernen und Kreativität führt, wird zu einer positiveren Haltung gegenüber Fehlern gelangen.

Wesentliches Merkmal einer positiven Fehlerkultur ist für viele, dass Fehler als ein "integrativer Bestandteil des Lernprozesses» angesehen und "nicht vermieden oder negativ bewertet» werden, vielmehr "erlaubt» sind: "An einigen Stellen im Lernprozess wird sogar dazu ermutigt, an Fehlern wird gearbeitet, der Umgang mit ihnen geübt» (Spychiger, Oser, Hascher \& Mahler, 1999, S. 44). Eine positive und konstruktive Fehlerkultur hängt für viele mit einem subjekt- bzw. lernendenorientierten Lern- und Lehrverständnis zusammen, einer Unterrichtskultur, die Entscheidungsoffenheiten schafft und individuelles entdeckendes Lernen auf eigenen Wegen ermöglicht (vgl. statt Anderer auch Wiater, 2004, S. 7). Fehler werden hier zu Einschätzungs-Helfern darüber, wo sich die Lernenden im Lernprozess befinden und wie sie am besten in ihren Entwicklungen unterstützt werden können. Für Lorenz ist solches Unterrichten ein «diagnostisches Unterrichten», welches auf dem Prinzip basiert,

die systematischen Fehler von Schülern zu identifizieren, den Unterricht auf diese Fehler zu fokussieren und damit in der Folge das Schülerverständnis für den Lerninhalt zu verbessern. Fehler werden dabei nicht tabuisiert oder aus dem Unterricht möglichst ausgeklammert aufgrund der Befürchtung, sie würden verwirren oder Falsches sich gar einschleichen. Diese Sicht führt Schüler dazu, im Laufe der Schulzeit Angst vor Fehlern zu entwickeln. Sinnvoller ist es hingegen, Schüler beim Experimentieren und damit beim Fehlermachen zu unterstützen, um diese Fehler in der Klasse zu diskutieren. Hierzu bedarf es eines Klimas in der Klasse, das durch wechselseitigen Respekt charakterisiert ist. (2004, S. 47f.; Hervorhebung von B. B.) 
Es gibt zahlreiche Beispiele dafür, wie Fehler im Unterricht nicht als persönliches Defizit aufgefasst, sondern als Arbeits- und Forschungsauftrag verstanden werden können, wie z. B. das Konzept «Von der persönlichen Grammatik zur Klassengrammatik» von Ruf, Frei und Zimmermann (2004) oder das MathematikSpiel «Wo steckt der Fehler?», über das Lorenz berichtet (2004, S. 49).

Die «konstruktive Hinwendung zu den Fehlern» soll ein «Lernen durch Verstehen» begünstigen (Edelstein, 1999, S. 126). "Fehler, Missverständnisse, Brüche können, indem ihre Genese aufgedeckt wird, zum Ausgangspunkt tieferen Verständnisses werden, können sozusagen ins Produktive gewendet werden» (Winter, 1995, S. 42). Schon bei Weimaer kann man lesen: «Sodann aber gibt es kaum einen lehrreicheren Weg zum Richtigmachen als den über das Falsche» (1939, S. 284); und weiter:

Was in unserem Zusammenhang dem Falschmachen ein besonderes Gewicht verleiht, ist die Tatsache, dass an ihm die Bedeutung des Richtigen erst in vollem Masse erlebt wird. Mit dem Innewerden des Falschen und der Einsicht in seine Unbrauchbarkeit und Wertlosigkeit erwachen wir zu kritischem Verhalten und erwacht in uns zugleich das Bedürfnis nach Richtigstellung. Einem solchen Erleben ist schon der Lernanfänger zugänglich. (1939, S. 286)

Fritz Oser hat solche Überlegungen, die sich auf die Kurzformel bringen lassen, «dass man besser weiss, was richtig ist, wenn man auch richtig weiss, was falsch ist» (Spychiger et al., 1999, S. 44), in seiner Theorie des «negativen Wissens» entfaltet. Wichtig dabei ist, den Ausdruck «negativ» «nicht etwa im moralischwertenden Sinne zu verstehen, nicht einmal im Sinne des 'Unerwünschten', sondern als Ergänzung oder Gegenstück zu 'positiv'»(Oser, Hascher \& Spychiger, 1999, S. 17). Andererseits ist zu beachten: "Nicht jede Fehlersituation ist eine gute Lernsituation; Fehlersituationen können auch unproduktiv oder ineffzient sein. .... Unproduktive und ineffiziente Fehlersituationen sollen nach Möglichkeit nicht auftreten» (Spychiger et al., 1999, S. 47); und weiter: "Das Fehlermachen und der richtige Umgang damit soll nie zum Selbstzweck werden, sondern immer den Lernprozess und die Unterrichtsqualität optimieren» (a. a. O., S. 62).

\section{"Gute Fehler» - "Schlechte Fehler»? \\ Ambivalenzen und Herausforderungen eines konstruktiven Umgangs mit Fehlern}

Auch wenn Oser et al. explizit die negativen Konnotationen des «negativen Wissens» negieren, weist dieser Terminus auf eine Ambivalenz in der positiven Bewertung des Falschen hin, auf die im Folgenden unter Berücksichtigung der Unterscheidung zwischen "guten» und «schlechten Fehlern» näher eingegangen werden soll. Selbst wenn Fehler, wie z. B. von Weimer, einerseits als wertvoll im Lernprozess geschätzt werden und man sich der Überzeugung anschliesst, dass sich in Wissenschaft und Technik «der Umweg über die Korrektur als der erfolg- 
reichste zur Lösung der jeweiligen Probleme erwiesen habe» (1939, S. 284), wird dies andererseits auch eingeschränkt:

Der Weg über das Falsche ist glücklicherweise nicht der einzige Weg zum Richtigen. Er sollte auch nicht der normale, der häufigst beschrittene sein. Jedenfalls schätzt man in der Schule wie in Beruf und Leben denjenigen Menschen am höchsten, der das, was er zu tun hat, von vornherein möglichst ohne Hapern und Stolpern zustande bringt. (1939, S. 287; Hervorhebung von B. B.)

Hat so gesehen das "Falsche» nur dann ein besonderes Gewicht für die Erkenntnis des «Richtigen» (s. Weimer, 1939, S. 286), wenn man das «Richtige» nicht auf Anhieb «richtig» macht? Stünde eine solche Einschätzung aber nicht im Gegensatz zu allen Auffassungen, die betonen, dass erst durch die konstruktive Auseinandersetzung mit Fehlern ein vertieftes Verständnis erreicht werden kann? Lässt sich dieser Gegensatz mit Oser et al. dadurch auflösen, dass Wissen um Falsches (negatives Wissen) «nicht bei jeder Form des Lernens von gleich grosser Bedeutung ist» (1999, S. 17), womit sich die Forschungsherausforderung stellen würde herauszufinden, in welchen Lern- und Lehrzusammenhängen (und allgemeiner in welchen Lebenszusammenhängen) «Fehlerwissen» relevant ist?

Denkt man bei dysfunktionalen Fehlern und Fehlersituationen, in denen nichts gelernt werden kann, an Fehler von Lehrenden «als Folge von mangelhaften und ungenauen Instruktionen oder schlechter Vorbereitung der Lehrperson», dann scheint es unproblematisch, diese als «schlechte Fehler» aus einer «Fehlerkultur» auszugrenzen und zu vermeiden (Oser et al., 1999, S. 24). ${ }^{3}$ Denkt man jedoch an «schlechte» Fehler der Lernenden, wird es schwieriger. Ein Beispiel von Wertheimer soll dies verdeutlichen:

Sie fragen einen Schüler

1. $12=3$ mal wieviel? Antwort: 4 .

2. $56=7$ mal wieviel? Antwort: 8 .

3. $45=6$ mal wieviel?

Angenommen, der Schüler würde als Antwort auf die dritte Frage sagen: "Sieben». Und wenn Sie ihn nach dem Grund fragten, würde er antworten: "Ist das nicht klar? Die vierte Zahl ist eins höher als die dritte». (1964, S. 34)

Während für Wertheimer hier ein Beispiel für eine "törichte», weil strukturblinde, Hypothese vorliegt, vertritt dagegen Kurt Reusser die These, dass diese Hypothese "nicht völlig strukturblind» und als "selbst generierte» Hypothese doch wertvoll für den Lernprozess sei (1999, S. 222). Reusser hält Wertheimers "Begriff der 'törichten Hypothese'» aus mehreren Gründen für «unglücklich»,

- weil er Lehrpersonen dazu verleitet, sehr früh in Denkprozesse evaluierend und zensierend einzugreifen;

- weil er dazu beiträgt, das im Zuge der Hypothesen-Generierung natürlicherweise entstehende Spiel der Phantasie ... zu hemmen;

- weil er den Standpunkt des Lehrer markiert, der immer schon zu wissen glaubt, welche Hypothesen in Sackgassen und welche zum Erfolg führen;

- weil er unterstellt, vom Standpunkt des Lernenden aus müsste in der Regel von 
Anfang an sichtbar sein, welche Hypothesen dumm sind und welche nicht. Gerade das ist aber meist nicht der Fall. Kreative Problemlösungen gehen nicht selten von Vermutungen aus, welche zuerst als Fehler erscheinen;

- weil er gekoppelt ist mit einer idealisierten Sicht einsichtigen Problemlösens und mit einer Unterschätzung der Rolle von Versuch und Irrtum so wie des tastenden, manchmal auch relativ blind suchenden Denkens. (1999, S. 222f.)

Nun geht es in dieser Auseinandersetzung um Hypothesen, die «gut» oder «schlecht» sein mögen, und nicht explizit um Fehler. Die Argumente Reussers lassen sich aber auch für eine kritische Betrachtung der Unterscheidung von «guten» und «schlechten Fehlern» heranziehen. Greift man den Aspekt der lehrendenzentrierten Sichtweise aus Reussers Argumentation auf, dann lassen sich zwei Gedankenstränge anschliessen.

So mag man sich zum einen fragen, ob nicht auch beide Autoren Recht haben könnten, wenn sie an unterschiedliche Lernende denken würden. Man könnte Wertheimer vielleicht zustimmen, wenn sein Schüler schon das Vermögen hätte, die Aufgaben richtig zu lösen. Ein solcher Schüler könnte dann auch vergleichbar mit dem schlecht vorbereiteten Lehrer sein, dem «schlechte Fehler» unterlaufen. Andererseits könnte man Reusser Recht geben, wenn der Schüler noch nicht über das erforderliche Vermögen verfügte. Sein Fehler wäre ein «guter Fehler», weil dieser ihn weiterbringt. Das Beispiel zeigt, dass der Terminus «Fehler» für sehr Unterschiedliches verwendet wird und ohne nähere Beschreibung möglicherweise in die Irre führen kann. Zum anderen mag man mit Heid weiterfragen: Wer sollte eigentlich das «Subjekt der Bestimmung dessen, was als richtig anzuerkennen ist» sein, wenn konstruktiv mit Fehlern umgegangen werden soll (1999, S. 129)? Nach Heid sind in "Autoritätsbeziehungen ... Autoritäten die eigentlichen Subjekte der Konstitution eines Fehlers»; «Diese Zuständigkeitsaufteilung ist geeignet, die Entwicklung autonomer sachlicher und moralischer Urteilskompetenz zu behindern» (1999, S. 134):

Meine Hypothese ist nun - und als solche ist sie überprïfungsbedürftig! -, dass die pädagogische Fruchtbarkeit eines Fehlers unter anderem davon abhängt, wie weit der Adressat der Beurteilung Gelegenheit erhält, sich als Subjekt kompetenten Urteilens zu begreifen, zu entwickeln und zu betätigen. Element einer «Fehlerkultur» hätte also die Entwicklung jenes Diskurses zu sein, in dem die Diskursteilnehmer sich an der Bestimmung dessen einflussreich beteiligen, was aus welchen Gründen als richtig oder falsch beurteilt zu werden verdient. Erst diese Mitwirkung an Entscheidungen, die den Fehler konstituieren, begründet Kompetenz - Kompetenz im Sinne von Zuständigkeit und Verantwortlichkeit sowie schliesslich auch Kompetenz im Sinne der Fähigkeit, mit Fehlern verantwortlich und konstruktiv umzugehen. (1999, S. 135; Hervorhebung von B. B.)

Folgt man diesen Überlegungen, dann heisst das, dass in jeweiligen Situationen allererst zu klären ist, ob und was für ein Fehler vorliegt. Was für die einen ein Fehler ist, mag es für Andere nicht sein, und was die einen als «schlechten Fehler» beklagen, ist für andere vielleicht ein «guter Fehler», der sie in ihrem Den- 
ken und Handeln weiter bringt. Wie wichtig es wäre, eine reflexive Ebene im Umgang mit Fehlern bei der Gestaltung von Lern- und Lehrprozessen als Herausforderung für eine positive Fehlerkultur aufgreifen zu können, wird in Heids Fazit deutlich:

Wer «Fehler» sehen oder kennen oder beklagen lernt, der lernt damit nicht auch schon, sich unabhängig zu machen von denen, die Fehler definieren und überaus selektiv interpretieren. Er lernt auf Phänomene zu schauen, die das Fehlerhafte scheinbar objektiv repräsentieren, er wird dazu verführt, Fehler zu ontologisieren. Er lernt nicht notwendig, nach den Ursachen für Fehlerhaftes zu fragen. Er lernt damit nicht notwendig, nach dem Kriterium zu fragen, ohne dessen Geltung es keinen Fehler gibt. Und er lernt nicht, sich einflussreich an Diskursen zu beteiligen, die der Geltungsbegründung unentbehrlicher Beurteilungskriterien dienen. (1999, S. 135)

Die Entfaltung einer positiven Fehlerkultur in Heids Sinne erfordert einen genauen Blick auf das, was als Fehler in Lern- und Lehrprozessen bezeichnet wird, und ein Ausloten der Möglichkeiten, wie Lernende und Lehrende sich über sie verständigen können. Im Folgenden soll an einigen Beispielen aus dem Alltag von Grundschulunterricht ein Horizont für mögliche Bestimmungen und Verständigungen erörtert werden. Die gewählten Beispiele sind einfach gehalten, so dass man sie auch als Einstieg nutzen kann, um mit den Kindern eine «Fehlersammlung» aufzubauen, die Grundlage für reflexive Klärungs- und Verständigungsprozesse ist. Dass dies keine künstlich-didaktische Aufgabenstellung, sondern eine «echte» Forschungsaufgabe ist - einmal abgesehen davon, dass es auch als reproduktive Arbeit für die Kinder eine Forschungsaufgabe bliebe -, wird bei einem Blick in die Literatur schnell ersichtlich. Reason hat den Klärungsbedarf zusammenfassend eingeschätzt:

There is no universally agreed classification of human error, nor is there one in prospect. A taxonomy is usually made for a specific purpose, and no single scheme is likely to satisfy all needs. Nearly everyone who has published in this field has devised some form of error classification. Consequently, the literature abounds with such taxonomies, reflecting a variety of practical concerns and theoretical orientations and ranging from the highly task specific to broad statements of underlying error tendencies. (1990, S. 10)

Um den Horizont möglicher Abgrenzungen nur ansatzweise anzudeuten, ist auch ein Blick auf verwandte Termini aufschlussreich. Wann genau will man davon sprechen, dass ein «Fehler» vorliegt, und in welchen Fällen liegt etwas anderes vor, etwa ein "Irrtum», "Missgeschick», "Patzer», "Schnitzer», eine "Panne», ein «Ausrutscher», «Störfall», «Versäumnis» bzw. "Vergessen», «Murks» bzw. «Murkel», «Misslingen», «Scheitern», «Versagen», «Unglück», «Fehltritt» «Fehlverhalten», eine blosse "Abweichung», "Differenz», "Störung» oder gar eine «Sünde», ein «Rechtsbruch», eine "Regelverletzung», "Täuschung», «Irreführung», ein «Verstoss», "Verbrechen» usw.? 


\section{Zur Vielfalt von Fehlern - Beispiele aus dem Alltag von Grundschulunterricht und Vorschläge für reflexive begriffliche Klärungs- und Verständigungsprozesse}

Unterschiedliche Verwendungsweisen des Terminus "Fehler» Das, was als «nicht richtig» bzw. als Fehler in Schule und Unterricht bewertet wird, kann das Ergebnis unterschiedlicher Konstellationen sein, wie folgende Beispiele aus dem Alltag von Grundschulkindern veranschaulichen, die für die Diskussion teilweise variierend aufbereitet wurden. Die Beispiele werden als Ausgang zur Entfaltung einiger Fragen, Kriterien und Aspekte genommen, die zu beachten sind, wenn man einen aufgeklärteren reflexiven Umgang mit Fehlern anstrebt:

Falsch-ausgemalt: (a) Ein Kind will etwas ausmalen, ohne dabei über die Linien zu malen. Es hat noch wenig Übung hierin und malt immer wieder über die Linien, was es aber sofort bemerkt, sich ärgert und versucht zu beheben, indem es das Übermalte auszuradieren versucht, was aber auch nicht so recht gelingt. (b) Ein anderes Kind soll etwas ausmalen, ohne über die Linien zu kommen. Die Aufgabe ist für dieses Kind kein Problem, aber es hat keine Lust und malt immer wieder über die Linien hinweg. (c) Ein drittes Kind malt konzentriert und kompetent sein Bild aus. Als es fast fertig ist, wird es ungeschickter Weise und versehentlich von seiner Nachbarin angestossen, rutscht mit dem Stift ab und malt über die Linien.

Vom Ergebnis her bedacht lösen alle drei Kinder die selbst- bzw. fremdgesetzte Aufgabe nicht. Aber haben sie alle einen Fehler gemacht, auch wenn es sich vielleicht um unterschiedliche Fehlerarten handelt - etwa im ersten Fall um einen Fehler aus Unvermögen, wenn man denn aus Unvermögen heraus einen Fehler begehen kann, im zweiten Fall um einen Fehler aus Unlust und im dritten Fall um einen Fehler aufgrund eines nicht selbst verschuldeten Versehens? Der zweite Fall (b) lässt sich auch mit Heids Überlegung zur Frage nach dem Subjekt, das bestimmt, was ein Fehler ist, interpretieren. Für die Person, die dem Kind die Vorgabe gegeben hat, das Bild auszumalen, mag ein Fehler vorliegen. Das Kind hingegen hat sich vielleicht nur auf das Ausmalen eingelassen, um einer schwereren Bestrafung (wegen Arbeitsverweigerung) zu entgehen; es nimmt aber die Konsequenzen für das schlechte Ausmalen bewusst in Kauf. Möglicherweise versteht es dieses Verhalten sogar als kleinen stummen Protest nach dem Motto: "Das hast du nun davon, wenn du mich zum Ausmalen zwingst, obwohl ich zu einem solchen Kinderkram überhaupt keine Lust habe».

Elektrischer-Strom-Hypothese: Im Sachunterricht über den elektrischen Strom betrachtet ein 10jähriges Mädchen nachdenklich eine Glühlampe. Dann stellt sie die Vermutung auf, dass die Glühlampe leuchtet, weil die Drähte, die in sie hin- 
einführen, zwei verschiedene Stoffe transportieren, die in ihr gemischt werden und die Drähte zum Leuchten bringen. Wenig später findet sie ihre Hypothese widerlegt, denn mit ihr lässt sich eine Reihenschaltung nicht erklären.

War die nun widerlegte Hypothese ein Fehler? Das Mädchen gelangt zwar aufgrund fehlenden Wissens zu einer Hypothese, die nicht zutrifft. Diese Hypothese macht aber relativ zu ihrem bisherigen Wissen sehr viel Sinn, ist logisch denkbar und ist als Reaktionshypothese (Zweistoffhypothese) neben anderen möglichen Hypothesen erwägenswert. Sollte man solche Hypothesen «Fehler» nennen? Heisst «Lernen auf eigenen Wegen» nicht auch, Hypothesen zu bilden, diese zu prüfen und je nach Ergebnis weiterzuentwickeln oder zu verwerfen und neue Hypothesen aufzustellen?

Falsche-Rechtschreibung: (a) Ein Mädchen will in seiner Geschichte das ihm unbekannte Wort "Walnuss» schreiben. Wie "Nuss» geschrieben wird, weiss es. Dann erinnert es, dass es «Wal» schon schreiben kann. Es überlegt: Eine Walnuss ist eine grosse Nuss so wie der Wal ein grosses Tier ist. Es schreibt das neue Wort richtig. (b) Ein Junge will auch "Walnuss» schreiben. Auch er kennt bereits "Nuss» und auch er denkt an einen Zusammenhang zwischen Wal und Walnuss. Wie «Wal» geschrieben wird, weiss er im Unterschied zu dem Mädchen nicht. Er erinnert aber die Regel, nach der man beim Schreiben von einem langem «a» wie bei «Wal» häufig «ah» schreiben muss. Er schreibt das neue Wort falsch. (c) In einer anderen Klasse haben die Kinder in der Diskussion zum Thema «Eine neue Bürgermeisterin bzw. ein neuer Bürgermeister wird gewählt» das neue Wort «Wahlurne» kennengelernt. Ein Mädchen überlegt, dass man es wahrscheinlich "Walurne» schreiben muss, denn für die vielen Stimmzettel braucht man eine sehr grosse Urne und «Wal» schreibt man ja auch ohne «h». (d) Noch eine Klasse weiter schreiben die Kinder ein Diktat. Zu den bekannten und geübten Wörtern des Diktats gehört auch das Wort «Wal». Einige Kinder schreiben es mit «h».

Ist es nicht sehr undifferenziert, in allen Fällen dieses Beispiels, in denen es zu einer falschen Rechtschreibung kommt, gleichermassen von «Rechtschreibefehlern» zu sprechen? ${ }^{4}$ Vergleichbar mit dem Beispiel «Elektrischer-Strom-Hypothese» sind auch die Fehler der Kinder im «Falsche-Rechtschreibung»-Beispiel (b), (c) auf noch fehlendes Wissen zurückzuführen, die Überlegungen selbst aber durchaus sinnvoll.

Gewollte Strafe: Eine Lehrerin stellt klar, dass diejenigen, die beim Abschreiben eines Textes von der Tafel mehr als zwei Fehler machen, in der nächsten grossen Pause in der Klasse bleiben und alles noch einmal abschreiben müssen. Ein Schüler, der lieber in der Klasse bleiben möchte, nutzt die Gelegenheit und baut etliche Fehler in seine Abschrift ein. Zu seiner Freude muss er die nächste grosse Pause in der Klasse verbringen.

Ist hier dem Schüler oder / und nicht vielmehr der Lehrerin ein Fehler unterlaufen, weil sie die Täuschung des Schülers nicht gemerkt hat? Kann der getäu- 
schten Lehrerin aber eigentlich ein Fehler unterlaufen sein, wenn sie die Täuschung nicht zu durchschauen vermochte und folgerichtig handelte und den Schüler «bestrafte»? Und weiter gefragt: Kann das, was für die eine Person ein «Fehler» ist, für eine andere Person kein «Fehler» sein? Oder bleibt ein «Fehler» ein «Fehler», kann aber verschiedenes für die Einzelnen bedeuten?

Naschverbot: Ein Kind weiss genau, dass es nicht von den frisch gebackenen Plätzchen naschen soll. Die Lust, einige zu probieren, ist gross. In einem günstigen Moment steckt es sich einige in die Tasche, um sie dann mit Genuss an einem sicheren Ort zu verspeisen. Hinterher hat es zwar ein leicht schlechtes Gewissen, aber es hätte nicht auf die Nascherei verzichten mögen.

Kann man lustvoll und absichtlich «Fehler» begehen?5 Oder ist das Übertreten des Naschverbots vielleicht nur aus der Aussenperspektive, nicht aber für das Kind selbst ein «Fehler»?

Stadtralley: Bei einer Stadtralley sollen sich die Kinder einer 4. Grundschulklasse den ihnen unbekannten Ort, in den sie ihre Klassenfahrt gemacht haben, erschliessen. Die Kinder starten in kleinen Gruppen, ausgerüstet mit Karten und den zu lösenden Aufgaben und Hinweisen, wie sie laufen sollen. In der Schule hatten sie im Sachunterricht das Lesen von Karten kennengelernt, so dass sie für die Aufgabe gut vorbereitet waren. Was niemand weiss: Wegen einer Grossbaustelle kann die Route nicht so verlaufen wie geplant. Der Umweg führt aber in einen Bereich, der von der Karte nicht mehr erfasst ist. So kommt es, dass die Kinder an einer Wegegabelung nicht wissen, wie es weiter geht. Während die einen vorübergehende Passanten um Rat fragen und den richtigen Weg einschlagen (a), hat eine andere Gruppe Pech, denn sie erhält versehentlich eine falsche Auskunft (b). Eine dritte Gruppe fragt niemanden und erwischt glücklicherweise den richtigen Weg (c), während die vierte Gruppe niemanden fragt und sich verirrt (d). In der fünften Gruppe befindet sich ein Mädchen, welches von seinen Eltern eine grössere Karte mitbekommen hat. Die Kinder vertun sich jedoch und verwechseln eine Rechts- mit einer Linksabbiegung (e). Die Kinder einer sechsten Gruppe entdecken im Schaufenster einer Touristeninformation einen Stadtplan. Sie lesen ihn allerdings nicht richtig. Im Unterschied zur fünften Gruppe vertun sie sich bei der Ausführung allerdings noch einmal und gelangen ans Ziel (f).

Haben alle drei Gruppen, die sich verlaufen haben, einen «Fehler» begangen? Und umgekehrt gefragt: Inwiefern sind die Gruppen, die das Ziel erreicht haben, «fehlerfrei» geblieben? Kann man, wenn man etwas nicht weiss und auch nicht wissen kann, hierum vielleicht reflexiv sogar weiss (Wissen um Nicht-Wissen), überhaupt einen «Fehler» begehen? Oder macht erst Wissen bzw. zumindest ein wenig Wissen «Fehler» möglich? 


\section{Vorschläge für reflexive begriffliche Klärungs- und Verständigungsprozesse}

Die angeführten Beispiele für unterschiedliche Verwendungsweisen des Terminus «Fehler», die sich auch gut mit Grundschulkindern weiter variieren und diskutieren lassen, regen an, das Spektrum möglicher Verwendungsweisen zu erschliessen und damit kompetenter im Umgang mit «Fehlern» zu werden. Ein weites Forschungsfeld wird erschlossen, wenn man erwägungsorientiert vorgeht und die jeweils denkbaren Möglichkeiten zu erfassen versucht.

- Es ist zu unterscheiden, ob eine Person das potenzielle Vermögen (die Fähigkeiten) gehabt oder nicht gehabt hätte, den «Fehler» zu vermeiden. ${ }^{6}$ Unter "potenziellem Vermögen» sollen hier kognitive Konzepte und handlungspraktische Erfahrungen begriffen werden. Die kognitiven Konzepte mögen z. B. für die Lösung einer Aufgabe adäquat oder nicht adäquat, die handlungspraktischen Erfahrungen mit den Konzepten gross oder gering sein. Das potenzielle Vermögen ist dasjenige Vermögen, über das jemand unter idealen Bedingungen verfügt. Das potenzielle Vermögen kann in jeweiligen Situationen nicht immer eingesetzt werden und beschränkt (gemindert) sein.

Mit Blick auf die Beispiele lassen sich damit «Fehler», die vermutlich typisch sind für entdeckendes Lernen auf eigenen Wegen, bei denen jeweiliges Vermögen allererst aufgebaut wird, wie im Falle des «Elektrische-Strom-Hypothese» Beispiels, und auch übendes Schulen von Fähigkeiten, wie im Falle des «Falschausgemalt»-Beispiels (a), unterscheiden von solchen «Fehlern», bei denen Wissen und "Vermögen» eigentlich vorhanden sind (bzw. sein könnten), wie etwa im «Falsch-ausgemalt»-Beispiel (b), im «Falsche-Rechtschreibung»-Beispiel (d) oder im «Stadtralley»-Beispiel (e). ${ }^{7}$

Folgende Erwägungstabelle ist eine Möglichkeit, die diesbezüglich systematisch denkbaren Fälle zusammenzustellen und sie regt zu weiteren Fragen und Präzisierungen an:

Erwägungstabelle 1: Zur Unterscheidung von «Fehlern» mit Blick auf unterschiedliche Vermögen.

\begin{tabular}{|l|l|l|l|}
\hline & $\begin{array}{l}\text { kognitive Konzepte, } z . \text { B. } \\
\text { zur Lösung einer Aufgabe }\end{array}$ & $\begin{array}{l}\text { handlungspraktische } \\
\text { Erfahrung, } z . \text { B. zur } \\
\text { Lösung einer Aufgabe }\end{array}$ & $\begin{array}{l}\text { Mögliche Beispiele für die } \\
\text { einzelnen Zeilen }\end{array}$ \\
\hline 1. Zeile: & sind adäquat & ist groß & s. Text \\
\hline 2. Zeile: & sind adäquat & ist gering & «Falsch-ausgemalt»-Beispiel (a) \\
\hline 3. Zeile: & sind nicht adäquat & ist groß & s. Text \\
\hline 4. Zeile: & sind nicht adäquat & ist gering & «Elektrische-Strom-Hypothese» \\
\hline
\end{tabular}

Die 1. Zeile wirft z. B. die Frage auf, was geschehen sein könnte, wenn einer Person trotz adäquaten Vermögens ein «Fehler» unterläuft. Es könnte sich in sol- 
chen Fällen etwa um sogenannte "Flüchtigkeitsfehler» handeln oder Fälle, bei denen das potenzielle Vermögen eingeschränkt ist, etwa aufgrund von Ablenkungen, Übermüdung oder Erschöpfung. ${ }^{8}$ Hinsichtlich der obigen Beispiele erinnert diese Zeile auch an das «Falsch-ausgemalt»-Beispiel (c), wo jemand alles richtig macht, es aber durch einen nicht selbst verursachten Zwischenfall zu einem «Fehler» kommt. Hinsichtlich der 3. Zeile stellt sich die Frage, ob solch ein Fall überhaupt vorliegen kann. Ein Beispiel wäre hier vielleicht, dass Schülerinnen und Schüler schon viel handlungspraktische Erfahrung mit elektrischen Schaltkreisen haben (etwa durch Arbeit mit entsprechenden Baukästen), hierfür jedoch über kein adäquates kognitives Konzept verfügen. Hinsichtlich eines nicht-adäquaten Konzeptes könnte man dabei weiter differenzieren und zwischen fehlenden und falschen Konzepten unterscheiden. Deutlich wird hier auch, dass das Verhältnis zwischen kognitiven Konzepten und handlungspraktischer Erfahrung näher und genauer zu bestimmen ist. Unterscheidet man zwischen erforderlichem Vermögen und vorhandenem potenziellem Vermögen zur Lösung einer Aufgabe, so ist weiterhin darauf zu achten, wie eine Person mit ihrem vorhandenen Vermögen umgeht, wenn ihr das erforderliche Vermögen (noch) fehlt. Geht sie adäquat mit ihm um und nutzt ihr vorhandenes potenzielles Vermögen gut, so mag sie zu sinnvollen, aber dennoch falschen Schlussfolgerungen gelangen, wie etwa der Reaktionshypothese (Zweistoffhypothese) im «Elektrische-Strom-Hypothese»-Beispiel (s. auch Erwägungstabelle 2).

- Es ist zu unterscheiden, ob eine Person ihr vorhandenes potenzielles Vermögen, unabhängig davon, ob es zur Problemlösung hinreicht oder nicht, einsetzt oder nicht einsetzt, und ob der Einsatz ihres Vermögens bewusst und absichtlich oder nicht bewusst und nicht absichtlich erfolgt.

\section{Erwägungstabelle 2: Zum unterschiedlichen Einsatz des vorhandenen potenziellen} Vermögens.

\begin{tabular}{|c|c|c|c|}
\hline & $\begin{array}{l}\text { vorhandene potenzielle } \\
\text { Vermögen }\end{array}$ & $\begin{array}{l}\text { Art des Einsatzes bzw. } \\
\text { Nicht-Einsatzes }\end{array}$ & $\begin{array}{l}\text { Mögliche Beispiele für die } \\
\text { einzelnen Zeilen }\end{array}$ \\
\hline 1. Zeile: & wird eingesetzt & ist bewusst / absichtlich & $\begin{array}{l}\text { "Elektrische-Strom-Hypothese», } \\
\text { "Falsche-Rechtchreibung ( (b), (c) }\end{array}$ \\
\hline 2. Zeile: & wird eingesetzt & $\begin{array}{l}\text { ist nicht bewusst / } \\
\text { nicht absichtlich }\end{array}$ & s. Text \\
\hline 3. Zeile: & wird nicht eingesetzt & ist bewusst / absichtlich & "Gewollte-Strafe«? s. Text \\
\hline 4. Zeile: & wird nicht eingesetzt & $\begin{array}{l}\text { ist nicht bewusst / } \\
\text { nicht absichtlich }\end{array}$ & "Stadtralley« (d) \\
\hline
\end{tabular}

Auch hier macht eine systematische Zusammenstellung auf zu klärende Fragen aufmerksam. Bei der 2. Zeile, die für diejenigen Fälle steht, in denen das vorhandene potenzielle Vermögen schon so gewohnheitsmässig eingesetzt wird, dass es dann nicht mehr aktiv bewusst wahrgenommen wird, stellt sich die Frage, was 
vorfallen muss, wenn es in diesen Fällen zu einem «Fehler» kommt. Die Zeile erinnert an die Konstellation der 1. Zeile der Erwägungstabelle 1. Ergänzend könnte man mit der Erwägungstabelle 2 hinzufügend vermuten, dass hier dann «Fehler» auftreten, wenn das vorhandene potenzielle Vermögen nicht ausreicht zur Lösung eines Problems oder einer Aufgabe. Bei der Interpretation der 3. Zeile mag man zunächst an das "Gewollte-Strafe»-Beispiel denken, wo ein Schüler absichtlich «Fehler» in eine Abschrift einbaut. Die Frage ist, ob man in diesem Fall sagen kann, dass er sein Vermögen nicht einsetzt. Aus der Aussenperspektive der Lehrerin, die weiss, dass er es eigentlich kann, mag das so aussehen. Für den Schüler selbst bedeutet das absichtliche Einbauen von «Fehlern» aber ja, dass er sein Vermögen sehr wohl einsetzt, nur eben nicht zur «Fehlervermeidung» 9 . Insofern fordert diese Erwägungstabelle dazu heraus, genauer zu werden. Eine Person, die absichtlich und bewusst ihr Vermögen nicht einsetzt, ist wohl eher jemand, "die sich keine Mühe gibt», "die es darauf ankommen lässt», «Fehler» zu begehen - wie man vielleicht das «Naschverbot»-Beispiel interpretieren könnte -, der mögliche «Fehler» gleichgültig sind oder die sich absichtlich "hilflos stellt», aus welchen Gründen auch immer.

- Es ist zu unterscheiden, ob eine Person sich selbst ursächlich einen «Fehler» zurechnet und / oder von Anderen zugerechnet bekommt:

\section{Erwägungstabelle 3: Zur Fehlerzurechnung.}

\begin{tabular}{|l|l|l|}
\hline & Person A rechnet sich & Person B rechnet \\
\hline 1. Zeile: & «Fehler» selbst zu & Person A «Fehler» zu \\
\hline 2. Zeile: & «Fehler» selbst zu & Person A «Fehler» nicht zu \\
\hline 3. Zeile: & «Fehler» nicht selbst zu & Person A «Fehler» zu \\
\hline 4. Zeile: & «Fehler» nicht selbst zu & Person A «Fehler» nicht zu \\
\hline
\end{tabular}

Diese Unterscheidungsdimension lässt sich an vielen der Beispiele durchspielen. So können im «Falsch-Ausgemalt»-Beispiel (c) alle Zeilen vorkommen, je nachdem, wie das Kind und etwa seine Lehrerin die Situation wahrnehmen. Weiss die Lehrerin nicht, dass das Kind versehentlich von der Nachbarin angestossen wurde, wird sie anders urteilen, als wenn sie dies weiss. Die hier systematisch zu bedenkenden vier Möglichkeiten lassen sich weiter dahingehend differenzieren, ob die jeweiligen Zurechnungen angemessen oder nicht angemessen sind. Diese Feststellung mag entweder von den Beteiligten selbst, also den Personen A und B (z. B. Schülerin und Lehrerin), oder einer dritten Person (z. B. Elternteil) getroffen werden, womit sich die Anzahl möglicher Konstellationen allein für das Zurechnen eines «Fehlers» auf 32 Möglichkeiten erhöht, auch wenn vermutlich nicht alle denkbaren Möglichkeiten sinnvoll sein werden, was hier aber nicht weiter erörtert werden kann. 
- Es ist zu unterscheiden, ob «Fehler» im Ergebnis einer Aufgabe und / oder auf dem Weg zur Lösung oder auch Realisierung einer Aufgabe bzw. eines Problems vorliegen. Auch hier sind zunächst wieder vier mögliche Konstellationen denkbar. Das «Stadtralley»-Beispiel (f) ist ein Beispiel für die Problemlage, dass ein Ergebnis «richtig» sein mag, ein Blick auf den Weg dorthin jedoch zeigt, dass dieses Ergebnis nur zufällig «richtig» und auf einer Kette von «Fehlern» beruht. Ein reflexives Wissen um eine solche «Fehler-Möglichkeiten» macht vorsichtiger im Umgang mit scheinbar «funktionierenden» oder «richtigen» Lösungen bzw. Realisierungen und ist grundlegend für die Entwicklung von Fehlersensibilität in einer «erwägungsorientierten Fehleraufsuchdidaktik».

Die hier nur andeutbaren Differenzierungen zu dem, was als «Fehler» verstanden wird, lassen ahnen, welche reflexiven Kompetenzen Lernende und Lehrende in einer «Fehlerkultur» entwickeln müssten, in der konstruktiv mit «Fehlern» umgegangen wird. Die Frage ist, ob man die Unterschiedlichkeit von «Fehlern» als «Fehlerarten» auffassen möchte oder - ergänzend neben der Unterscheidung von «Fehlerarten» - in manchen Fällen andere Termini verwenden möchte. Konsequenz einer Unterscheidung etwa von «Misslingen», wie im «Falsch-ausgemalt»-Beispiel (a), von «Irrtum», 10 wie im «Falsche-Rechtschreibung»-Beispiel (b), (c) oder im «Elektrische-Strom-Hypothese»-Beispiel, von "Täuschung», wo absichtlich ein «Fehler» begangen wird, wie im "GewollteStrafe»-Beispiel, ${ }^{11}$ oder von "Scheitern», was nicht selbst, sondern aufgrund etwa äusserer Umstände verursacht wurde, wie im «Falsch-ausgemalt»-Beispiel (c), wäre, dass man vermutlich nur noch in wenigen Fällen von «Fehlern» sprechen könnte. Nach den hier vorgestellten Überlegungen könnte ein wesentliches Kriterium für solche dann «echten Fehler» vielleicht das potenzielle Vermögen der Fehlerbegehenden sein, das aber wie angedeutet noch näher zu spezifizieren wäre. In diesem Sinne würde etwa das Kind im «Falsch-ausgemalt»-Beispiel (b), welches das Vermögen zum richtigen Ausmalen hat, einen "Fehler» begehen. Als eine Arbeitsdefinition, die «Fehler» in diesem Sinne z. B. von «Misslingen», «Irrtum», «Täuschung» oder «Scheitern» ermöglichte, liesse sich aus den vorangegangenen Erörterungen vorerst festhalten: Ein Fehler ist eine Soll-Ist-Differenz, die ursächlich einem oder mehreren gedächtnisfähigen Wesen zugesprochen wird und (gewissen) Intentionen sowie dem potenziellem Vermögen der gedächtnisfähigen Wesen zuwiderläuft. ${ }^{12}$

Wie immer man sich hinsichtlich der Bezeichnung von «Fehlern» entscheiden mag, es bleibt die Herausforderung, kompetent mit den exemplarisch dargelegten Unterschieden umzugehen und einen konstruktiven Umgang mit «Fehlern» in Lern- und Lehrprozessen zu fördern. Wie hierbei eine «erwägungsorientierte Fehleraufsuchdidaktik» unterstützen könnte, soll im letzten Abschnitt skizziert werden. Die vorangegangenen Klärungsvorschläge lassen sich als ein Beispiel für einen erwägungsorientierten Umgang bei der Begriffsklärung verstehen, wobei die eingeführten Erwägungstabellen weiter zu spezifizieren und insbesondere auch in ihren Bezügen untereinander zu klären wären, was eine 
Voraussetzung für klärungsförderliche empirische Untersuchungen wäre. Herauszuarbeiten wären weiterhin die jeweiligen Grenzen dessen, was erwogen wurde oder erwogen werden kann, wobei kombinatorische Zusammenstellungen wie Erwägungstabellen insofern hilfreich sind, weil sie durch die Offenlegung des Erwogenen gut kritisierbar sind und Nicht-Erwogenes oder Grenzen jeweiligen Wissens leichter angeben lassen.

\section{überlegungen zur Entwicklung einer erwägungs- orientierten Fehleraufsuchdidaktik}

\section{Zum Konzept einer Erwägungsorientierung}

Gegenstand des Erwägungskonzeptes sind Entscheidungen, einschliesslich reflexiver Entscheidungen, etwa bezüglich bestimmter Problemlagen oder Fragestellungen nicht oder nicht mehr selbst zu entscheiden. Wird keine Entscheidung (mehr) getroffen, so mag man fremden Vorgaben, die das Ergebnis von Entscheidungen Anderer sein mögen, oder eigenen Gewohnheiten bzw. Routinen als selbstgesetzten Vorgaben folgen. Dem Erwägungskonzept liegt ein weit gefasstes Entscheidungsverständnis zugrunde. Unter einer «Entscheidung» wird das Erwägen von mindestens einer Möglichkeit sowie die positive oder auch negative Bewertung des Erwogenen verstanden. Erwägen und Bewerten werden als Überlegen in Zusammenhang mit einem Auswahlgedanken (z. B. eine Frage, Problem, Aufgabe, Zielsetzung) gesehen. Zentral für die Haltung gegenüber «Fehlern» bzw. «Falschem» ist, dass vom Erwägungskonzept her zwischen Erwägungsalternativen und Erwägungsebene sowie Lösungsalternativen und Lösungs- und Realisierungsebene unterschieden wird. Erwägungsalternativen sind diejenigen Alternativen, die in einem Entscheidungszusammenhang erwogen werden. Lösungsalternativen sind diejenigen Alternativen, die aus verschiedenen Entscheidungszusammenhängen (Erwägungen und Bewertungen) hervorgegangen sind und auf der Lösungsebene von denselben Personen zu unterschiedlichen Zeiten oder von verschiedenen Personen vertreten und / oder realisiert worden sind. Dabei kann das, was für eine Person bereits eine Lösung ist, mit der sie etwa im Widerstreit zu einer anderen Person steht, die eine andere Lösung vertritt, für eine dritte Person allererst zu erwägen sein. So muss eine Richterin beispielsweise die Lösungsalternativen zweier streitender Parteien ihrerseits als zu erwägende Alternativen bedenken, dabei weitere denkbare Lösungsmöglichkeiten erwägen und bewerten, um dann zu ihrem Urteil (ihrer Entscheidung) zu gelangen, welche für die streitenden Parteien eine Lösungs-Vorgabe ist, der sie zu folgen haben.

Kerngedanke des Erwägungskonzeptes ist nun, dass die Kontravalenz, die zwischen Lösungsalternativen bestehen muss - wenn ich mich für den linken Weg entschieden habe, dann kann ich nicht den rechten Weg zur gleichen Zeit selbst gehen -, auf der Erwägungsebene nicht nur nicht besteht, sondern es hier 
im Gegenteil sinnvoll ist, eine möglichst umfassende Integration aller problemadäquat zu erwägenden Alternativen, die einander auch widersprechen mögen, anzustreben, jedenfalls dann, wenn man zu gut begründeten und verantwortbaren Lösungen gelangen möchte. Erwogene Alternativen werden damit zu einem Geltungsbezug für die Qualität von jeweiligen Lösungen und sie sind insofern zu bewahren. Sie haben über ihre Bedeutung im "context of discovery» eine Bedeutung im "context of justification». Denn nur dann, wenn man um die möglichen Alternativen weiss und diese problemadäquat (vollständig) anzugeben vermag, mag man für eine bevorzugte Lösungsmöglichkeit hoffen, sie mit hinreichenden Gründen verteidigen zu können. Mit der Erwägungsgeltungsbedingung unterscheidet sich das Erwägungskonzept in seiner Wissenschaftsauffassung vom Umgang mit qualitativen Alternativen von bisherigen Traditionen. ${ }^{13}$ Ein Wissen um zu erwägende Möglichkeiten lässt auch zu, dass man nunmehr mit Gründen angeben kann, mehrere Lösungsmöglichkeiten seien "gleichwertig» und zwischen ihnen sei nicht mit Gründen zu entscheiden, somit also eine dezisionäre Konstellation vorliege. Nun ist es weder möglich noch sinnvoll, immer und überall umfassend zu erwägen. Erwägungsorientierung kann ein Denken und Handeln reflexiv aber auch gerade in solchen Konstellationen leiten, wo nicht erwogen werden kann, und sei es, dass es vorsichtiger macht und man sich im Wissen um mangelnde Begründbarkeit korrekturinteressierter verhält.

Einen Eindruck davon, was dies für reflexive Verständigungs- und Klärungsprozesse im Umgang mit «Fehlern» bedeuten kann, sollten die Beispiele und ihre Erörterung im vorangegangenen Abschnitt geben. Der Vorteil derartigen Vorgehens ist, dass man sich über Unterschiede und Gemeinsamkeiten verschiedener bisheriger Verständnisse austauscht und versucht, weitere mögliche Auffassungen zu erschliessen, wodurch jeweilige Subjektivität entfaltet und eine Grundlage für Verständigung geschaffen wird. In einem Erwägungshorizont möglicher Verwendungsweisen des Terminus «Fehler» kann jede und jeder die eigene Position im Wissen um alternative Positionen verorten, und wenn dies geschehen ist, mag man sich auch auf eine gemeinsame Sprachregelung einigen. Ist dies aufgrund unterschiedlicher Sprachgewohnheiten nicht möglich, so kann man sich mit Hilfe des erarbeiteten beispielsbezogenen Erwägungshorizontes dennoch verständigen, weil jeder und jede durch ihn weiss, wie was gemeint ist.

\section{Aktives Aufsuchen von Fehlern und Falschem aus erwägungsorientierter Perspektive}

Neben dem Nutzen von erwägungsorientierter Begriffsbildung und erwägungsorientiertem Vorgehen in reflexiven Klärungs- und Verständigungszusammenhängen, ist Erwägungsorientierung mit ihrer Erwägungsgeltungsbedingung auch für den Umgang mit jeweiligen konkreten «Fehlern» in Schule und im Unterricht hilfreich. Insofern die Erwägungsgeltungsbedingung die stärkste Begründung für jeweilige Lösungen immer dann gibt, wenn sie möglichst entfaltet ist und die problemadäquaten zu erwägenden Alternativen sich vollständig an- 
geben lassen, schliesst die Suche nach Alternativen auf der Erwägungsebene die Suche nach Alternativen ein, die von der Lösungs- und Realisierungsebene her bedacht als «falsch» oder «als Fehler» bewertet werden würden. Aus der Perspektive der Erwägungsebene sind dies - wie erläutert - jedoch keine «Fehler», sondern zu erwägende Alternativen, die helfen, die Begründungs- und Verantwortungsqualität der schliesslich gewählten Lösung einzuschätzen. Hier zeigt der erwägungsorientierte Fehleraufsuchansatz grosse Nähen insbesondere zu Osers Überlegungen zu einem konstruktiven Umgang mit Fehlern. Denn wie oben dargestellt, trägt bei Oser das negative Wissen, das Fehlerwissen, dazu bei, das positive Wissen zu stärken und auch Oser möchte, dass das negative Wissen in dieser Funktion nicht als negativ «im moralisch-wertenden Sinne» verstanden wird (Oser et al., 1999, S. 17). Vor allem die Auffassung, dass das «Richtige und Wahre ... seine Eindeutigkeit erst durch das Unrichtige» erhält (Oser et al., 1999, S. 18), scheint eine hohe Nähe zu dem Gedanken der Erwägungsgeltungsbedingung zu haben, wenn man etwa weiter liest, dass es für Oser et al.

für Wissenskonzepte nicht bloss wichtig ist, was sie bestimmt, sondern auch was sie ausgrenzt. Selbst einfache Bestimmungen z.B. eines Tieres sind nicht zu leisten, obne eine Vorstellung davon zu haben, was ein Vogel ist und was er nicht sein kann. Abgrenzungen schützen genau so vor Falschem, wie die tatsächlichen Bestimmungselemente an sich. Wir haben deshalb auch den Begriff des "Abgrenzungswissens" eingeführt. Wenn jemand z.B. das Konzept der "Demokratie» erarbeitet, dann sind auch jene inhaltlichen Bestandteile wichtig, die diesem Konzept entgegenstehen, hier etwa "Monarchie» oder "Totalitarismus». (a. a. O., S. 19)

Es lässt sich aus erwägungsorientierter Perspektive anknüpfen: Je umfassender es gelingt, methodisch geleitet alternative «Herrschaftsformen» zusammenzustellen - einmal angenommen, dass ,Herrschaftsform' der adäquate Oberbegriff wäre, zu dem seinerseits Alternativen zu erwägen wären, - um so besser wird die Geltungsbedingung dafür, mit Gründen eine dieser Formen als Lösung den anderen Lösungsmöglichkeiten vorziehen zu können.

Mit dem Erwägungskonzept lässt sich ein Zusammenhang zwischen Erwägungsgeltungsbedingung und damit Begründungsqualität sowie positivem Wissen herstellen, bei dem "positives Wissen» nicht nur durch «negatives Wissen» gestärkt wird, sondern ohne «negatives Wissen» als ein "positives Wissen» sogar fragwürdig ist. Konsequenz für eine «Fehlerkultur», die diese Zusammenhänge nutzen will, wäre aus erwägungsorientierter Perspektive die systematisch methodisch orientierte Entfaltung aller derjenigen subjekt- bzw. lernendenorientierten Ansätze, die den Umgang mit Alternativen (Hypothesen, Lösungswegen, Lösungen usw.) - wozu dann auch immer «Irrwege», "Sackgassen», «Misslingen», «Irrtümer» und «Fehler» gehören - konstitutiv für ein entdeckendes Lernen auf eigenen Wegen halten. Obwohl es hier viele Anknüpfungspunkte gibt, fehlt es an entfalteten qualitativen Methoden und Traditionen, die qualitative Wissensstände so aufbereiten, dass jeweiliges «Lösungswissen» eingebettet in «Erwägungswissen» forschend erarbeitet werden kann. Die Entfaltung von "positiver 
Fehlerkultur» muss aus erwägungsorientierter Perspektive mit der Entfaltung von mehr «Forschungskultur» einhergehen.

\section{Erwägungsorientierung und reflexiver Umgang mit Fehlern: Anknüpfungspunkte und Perspektiven}

Für viele Autorinnen und Autoren ist ein reflexiver Umgang mit Fehlern grundlegend für eine Fehlerkultur, in der aus Fehlern gelernt werden kann. Reflektiert wird, warum ein eingeschlagener Lösungsweg missglückte. Reflektiert wird weiterhin der Vergleich unterschiedlicher Ergebnisse und Lösungswege. So ist etwa für Ruf et al. es für die Arbeit an der «persönlichen» Grammatik wesentlich, dass die Schülerinnen und Schüler «Fehler wählen, aus denen sie etwas lernen können» (2004, S. 100), was selbst gelernt werden muss. Auch Lorenz betont, dass zu erklären ist,

wie der Fehler zustande kam, welcher fehlerhafte Denkprozess zugrunde lag. Dieser zweite Schritt ist wichtig. Lernprozesse werden initiiert, wenn die Schülerinnen und Schüler über Lösungswege nachdenken. Diese Reflexion macht den eigentlichen Lernfortschritt aus. Die eigenen Lösungsversuche müssen verglichen und bewertet werden. Kann es sein, dass in dem Lösungsversuch des anderen ein richtiger Kern steckt? Wieso gehe ich nicht ebenso vor? Welche Annahmen hat der andere getroffen? (2004, S. 49; Hervorhebung von B. B.)

Vor allem ist die von Heid aufgeworfene Fragestellung, wann etwas ein «Fehler» ist, für die in diesem Beitrag Arbeitsvorschläge gemacht wurden, zu lösen. Erwägungsorientierung sensibilisiert für solche Reflexionen und macht kompetenter im Umgang mit Nicht-Wissen und Grenzen des Wissens. Denn Erwägungsorientierung führt zu Fragen, wie: Kann das, was für dich ein «Fehler» ist, für mich kein «Fehler» sein? Wie sind wir zu dem, was «richtig» ist, gekommen? Sind alle verschiedenen Lösungswege, auf denen wir zu der «richtigen» Lösung gelangt sind, «richtig»? Woher wissen wir, ob das, was «richtig» ist, die einzige «richtige» Lösungsmöglichkeit ist? Können wir das überhaupt wissen? Wie gehen wir damit um, wenn wir dies nicht wissen?

Welche nicht nur methodisch-reflexiven Kompetenzen, sondern auch fachlichen Anforderungen dies an Lehrerinnen und Lehrer stellt, lässt sich etwa an dem «Elektrische-Strom-Hypothese»-Beispiel verdeutlichen. Lehrende müssen nicht nur erkennen können, dass die «Reaktionshypothese (Zweistoffhypothese)» des 10-jährigen Grundschulmädchens angesichts ihres Wissensstandes eine adäquate neben anderen möglichen Hypothesen, wie der «Transporthypothese», der "Kreislaufhypothese» oder der «Durchdringungshypothese» ist (s. hierzu Götz, Dahncke und Langensiepen (Hrsg.), 1992, S. 56), um diese Alternativen mit den Lernenden erwägen zu können, sie müssen auch wissen, wie mit welchen Experimenten - die Lernenden ihre erwogenen Hypothesen prüfen können.

Die in der Arbeit vorgeschlagenen Differenzierungen im Umgang mit «Fehlern» sind aus erwägungsdidaktischer Sicht eine erste Orientierung, wie sich 
Lehrende und Lernende von Anfang an ${ }^{14}$ anhand von erlebten «Fehlerbeispielen» aus ihrem schulischen und ausserschulischen Alltag zunehmend selbst ein «Fehlerwissen» erarbeiten könnten, durch das sie zunehmend reflexiv «fehlerkompetenter» werden würden. Voraussetzung für ein konkreteres schulisches erwägungsorientiertes Fehlerprojekt wäre zunächst eine entsprechende Aus- bzw. Fortbildung der Lehrerinnen und Lehrer zum reflexiven methodisch orientierten Umgang mit der Komplexität des Themas «Fehler». Sodann könnten etwa in einem fächerübergreifenden Fehlerforschungsprojekt, bei dem Lehrerinnen und Lehrer mit ihren Schülerinnen und Schülern gemeinsam «Fehler» kontinuierlich sammeln und auswerten, die ihnen im schulischen, aber auch ausserschulischen Alltag begegnen, insbesondere folgende Fragen untersucht werden:

- $\mathrm{Zu}$ welchen Bestimmungen und Klassifikationen gelangen die Lernenden und Lehrenden?

- Inwiefern wird im alltäglichen Umgang mit «Fehlern» auf das Fehlerforschungsprojekt Bezug genommen?

- Verändert sich der Umgang mit «Fehlern» im Verlaufe des Projektes?

Würde man die in dieser Arbeit angedeuteten Differenzierungen weiter verfolgen, so wäre eine Fehlerkultur durch eine entfaltete Fehleraufsuchdidaktik reflexiv eingebettet in eine Kultur des kompetenten Umgehens mit «Irrtümern", «Misslingen», «Scheitern» usw. Inwiefern derartige Fehlerkultur dann auch hilfreich sein könnte bei der Einschätzung und dem Vergleich von Lern- und Lehrszenarien, wäre in vergleichenden Folgeprojekten, die bereits eine gewisse Praxis erwägungsorientierter Fehleraufsuchdidaktik voraussetzen würden, näher $\mathrm{zu}$ erforschen: Wird in vorgabeorientierten Lern- und Lehrsituationen eine «Fehlerkultur» nur im eingeschränkten Sinne vorliegen können? Werden in entscheidungsorientierten Lern- und Lehrsituationen weniger «Fehler» als vielmehr «Irrtümer» und «Misslingen» zu diagnostizieren sein? Dies könnte schliesslich zu einer Erwägungskompetenz voraussetzenden Differentialdiagnostik führen. Wenn alle diese Fragen sowie die Herausforderung der Entwicklung einer entfalteten Fehleraufsuchdidaktik zu einem Forschungsprojekt nicht nur in den Schulen werden könnten, wären wir - so meine abschliessende Vermutung auch gesamtgesellschaftlich ein gutes Stück weiter in der Verwirklichung von reflexiver Fehlerkultur.

\section{Anmerkungen}

1 Zum Wandel dieser Einstellung im Fremdsprachenunterricht vgl. Knapp-Potthoff (1987, S. 207ff).

2 Vgl. auch Dewey (1980, S. 205). Es fällt auf, dass Dewey einmal «error», andere Male «mistake» schreibt. Zur Umstrittenheit der Verwendung von «error», «mistake», «slip» usw. vgl. den Bericht über zwei Konferenzen zum Thema «Human Error», zusammengefasst von Senders und Moray (1991, z. B. S. 14).

3 Die Frage ist jedoch, ob in einer entfalteten Fehlerkultur gerade mit Blick auf zukünftige Vermeidung derartiger «Lehrendenfehler» diese nicht auch näher analysiert werden sollten und z. B. gefragt werden müsste: Fehlt der Lehrperson grundsätzlich wegen einer schlech- 
ten Ausbildung das Vermögen, jeweilige «Lehrfehler» nicht zu begehen? Fehlt ihr zudem auch das reflexive Vermögen, ihr fehlendes Vermögen als Defizit zu erkennen und entsprechende Massnahmen zu ergreifen (Beratung mit Kolleginnen und Kollegen, Fortbildung usw.)? Oder ist ihr ihre Arbeit nicht hinreichend relevant und sie bereitet sich «nur» schlampig vor, könnte es eigentlich aber besser? Zur Frage, inwiefern man auch für NichtWissen verantwortlich ist und Nicht-Wissen keine Entschuldigung für «Fehler» ist, vgl. Weingardt (2004, S. 210ff). Vgl. auch Anmerkungen 6 und 8.

4 Zur unterschiedlichen «Bedeutung des Fehlermachens pro Lernphase» vgl. auch Spychiger et al.: "Die wichtige Unterscheidung von 'Fehler vor' und 'Fehler nach' dem Lernprozess führt zur Hypothese, dass eine sinnvolle Auseinandersetzung mit falschen, unsicheren oder problematischen Lösungswegen während des Lernprozesses zu weniger Fehlern führen kann» (1999, S. 15).

5 In diesem Sinne betont etwa Bucher: «Menschen begehen Fehler, oft im Stress und nicht gewollt, oft aber auch bewusst, mit Absicht, ja mit Lust» (1998, S. 615, Nr. 7). Vgl. dagegen die Auffassung, dass Fehler von den Fehlerbegehenden nicht-intendiert sind, z. B. von Kobi, s. Anmerkung 9.

6 In diesem Sinne gilt etwa für Frese und Zapf: «Man spricht nur dann von einem Fehler, wenn er potentiell vermeidbar gewesen ist» (1991, S. 15). Wo die Grenzen dessen liegen, was man jemanden bzw. was sich jemand selbst adäquat als Vermögen zurechnet, wäre allerdings im Einzelfall genauer zu klären und muss im Rahmen dieser Arbeit offen bleiben. Vgl. hierzu auch Anmerkung 8.

7 Hinsichtlich des Auftretens von Fehlern trotz potenziellen Wissen-Könnens und Vermögens vgl. auch Weingardt, der die Unterscheidung und Bewertung von Fehlern und Irrtümern einem historischen Wandel unterworfen sieht. Nach Weingardt werden die «Vorzeichen von Irrtum und Fehler, von Nicht-Wissen und Fehlhandeln ... tendenziell vertauscht» (2004, S. 213). So, wie man Fehler in einer Gesellschaft, in der eine "weitreichende Zugriffsfreiheit auf Information» (2004, S. 211) besteht, nicht mit Nicht-Wissen entschuldigen und als blosse Irrtümer hinstellen kann, so muss man andererseits berücksichtigen, dass die «heute potenzierte Informationsbeschaffung, -verarbeitung und -aktualisierung, die der Einzelne zu leisten hat, ... beinahe zwangsläufig mit gesteigerten Erinnerungsverlusten verbunden" ist (2004, S. 212). Zu unterscheiden ist also zwischen dem Vorliegen oder dem Fehlen von Zugriffsfreiheit auf Information und einem verantwortbaren Umgang mit Nicht-Wissen angesichts der Möglichkeit, vieles wissen zu können, aber kapazitativ eingeschränkt zu sein.

8 Das wirft die Frage nach dem reflexiven potenziellen Vermögen auf, mit dem eigenen potenziellen Vermögen verantwortbar umzugehen. Muss zu meinem potenziellen Vermögen, z. B. des Autofahrens, nicht auch das adäquate reflexive Einschätzungsvermögen dazugezählt werden, wann mein Autofahr-Vermögen eingeschränkt ist und ich es lieber lassen sollte? Der Ausdruck "potenzielles Vermögen» ist diesbezüglich weiter zu klären. Vgl. hierzu auch Anmerkungen 3 und 6.

9 Die Vermeidung von Fehlern gilt einigen als wichtiges Bestimmungsmerkmal. So sind etwa für Kobi Fehler "per se intentionswidrig» und unbeabsichtigt (1994, z. B. S. 6). Aber es gibt auch hier wieder andere Auffassungen, vgl. Anmerkung 5.

10 Für viele hat Irrtum im Unterschied zum Fehler etwas mit Nicht-Wissen und falschen Annahmen zu tun, so z. B. für Weimer (1929, S. 5; 1939, S. 284) oder Kobi (1994, S. 6). Interessant sind dabei die Konsequenzen, die Weimer aus seiner Unterscheidung zieht: «Das Irrtümliche ist verantwortungsfrei; für das Fehlerhafte hat der Schüler einzustehen. Dieser Grundsatz gilt für die ganze Schulzeit, ja er gilt auch für Beruf und Leben» (1939, S. 284). Anregend sind ausserdem Überlegungen von Mach, für den der «klar erkannte Irrtum ... als Korrektiv ebenso erkenntnisfördernd wie die positive Erkenntnis» ist (1980, S. 116) und «dieselben psychischen Funktionen, nach denselben Regeln ablaufend ... einmal zur Erkenntnis, 
das andere Mal zum Irrtum führen, und dass nur die wiederholte, sorgfältige, allseitige Prüfung uns vor letzterem schützen kann» (1980, S. 125; ähnlich vgl. auch Selz, 1922, S. 12).

11 Das «gewollte-Strafe»-Beispiel zeigt auch, dass die Täuschung des Schülers, wenn sie unerkannt bleibt, bei der Lehrerin wohl eher zu einem «Irrtum», denn zu einem «Fehler» führt, wenn man davon ausgehen würde, dass der Lehrerin hier hinreichendes Wissen fehlt und man vorhandenes bzw. fehlendes Wissen als Abgrenzung von «Irrtum» und «Fehler» nimmt (s. Anmerkung 10).

12 Es ist hier nicht der Raum, diesen Definitionsvorschlag näher zu erläutern. Drei Anmerkungen seien aber zur Erläuterung gemacht: 1.) Der Ausdruck «Soll-Ist-Differenz» wurde gewählt, weil sich unter dem zugehörigen relativ abstrakten Begriff unterschiedliche konkretere Beschreibungen in der Literatur fassen lassen. In der Literatur wird diese z. B. als Diskrepanz zwischen gesetztem Ziel und Nichterreichen dieses Ziels oder eines Teilziels beschrieben (vgl. statt Anderer Frese \& Zapf, 1991, S. 15), als «die Abweichung vom Richtigen (i.w.S.)» (Heid, 1999, S. 129), als «unerwünschte Normabweichung» (Kobi, 1994, S. 6) oder «als von einer Norm abweichende Sachverhalte oder von einer Norm abweichende Prozesse verstanden» (Oser et al., 1999, S. 11). Diese Soll-Ist-Differenz wird für Lern- und Lehrprozesse auch als Widerspruch beschrieben zwischen dem «Ergebnis einer Handlung» und «einer mehr oder weniger objektiven Richtigkeitsnorm oder einer mehr oder weniger subjektiven Erwartung der Erziehenden» (Haeberlin, 1999, S. 92). 2.) Die Rede von «gedächtnisfähigen Wesen» soll die Bestimmung von «Fehler» solange nicht auf Menschen beschränken, wie ungeklärt ist, ob man nicht auch bei Tieren Beispiele dafür hat, dass sie mit "Fehlern» umgehen (vgl. hierzu die Beschreibung einer Szene zwischen Schimpansenmutter und Tochter beim Erlernen des Nüsseknackens von Boesch 1991, S. 532). 3.) Dass Fehler nur «gewissen» Intentionen zuwiderlaufen können, will den Überlegungen von Bucher Rechnung tragen, nach denen es intrapsychisch durchaus widerstreitende Intentionen geben kann, wie im «Naschverbot»-Beispiel (vgl. auch Anmerkung 5).

13 Ausführlicher hierzu Blanck (2002), insbesondere Kap. I. Dazu, dass es im Unterschied zum Umgang mit qualitativen Alternativen beim Umgang mit quantitativen Alternativen entfaltete Forschungstraditionen gibt, die auch vermutlich den Erfolg der neuzeitlichen Wissenschaften ermöglicht haben, weil die Quantifizierungsmöglichkeiten «für jeweilige Bereiche alle überhaupt denkbaren Alternativen erwägen lassen» vgl. Loh (1992, S. 72, Nr. (21)). Zum Konzept einer Erwägungsorientierung vgl. auch zusammenfassend Blanck (2004).

14 Dazu, dass dies grundsätzlich auch schon mit Grundschulkindern möglich ist, vgl. Hartinger (1997), auch wenn dieser hier «nur» über einen fachspezifischen Umgang mit einer bestimmten Art von «Fehlern» berichtet, nämlich «Fehlern», die einem noch unterlaufen, weil man noch keine hinreichende Übung und Routine entwickelt hat.

\section{Literatur}

Blanck, B. (2002). Erwägungsorientierung, Entscheidung und Didaktik. Stuttgart: Lucius \& Lucius.

Blanck, B. (2004). Erwägungsorientierung. Information Philosophie, 32 (1), 42-47.

Boesch, C. (1991). Teaching among wild chimpanzees. Animal Behaviour 41 (3), 530-532.

Bucher, A. A. (1998). Warum aus Fehlern nicht gelernt wird? Konstruktiv-kritische Anmerkungen zu F. Osers Konzept der «negativen Moralität». Ethik und Sozialwissenschaften 9 (4), 614-616.

Dewey, J. (1978). How we think. In J. A. Boydston (Ed.), John Dewey. The Middle Works, 1899-1924. Volume 6: 1910-1911 (S. 177-356). Carbondale, Edwardsville: Southern Illinois University Press \& London, Amsterdam: Feffer \& Simons, Inc. 
Dewey, J. (1980). Democracy and Education. In J. A. Boydston (Ed.), John Dewey. The Middle Works, 1899-1924. Volume 9: 1916. Carbondale, Edwardsville: Southern Illinois University Press \& London, Amsterdam: Feffer \& Simons, Inc.

Edelstein, W. (1999). Aus Fehlern wird man klug. Zur Ontologie der Fehlertypen. In W. Althof (Hrsg.), Fehlerwelten. Vom Fehlermachen und Lernen aus Fehlern (S. 111-127). Opladen: Leske + Budrich.

Frese, M. \& Zapf, D. (1991). Fehlersystematik und Fehlerentstehung: Eine theoretische Einführung. In Dies. (Hrsg.), Fehler bei der Arbeit mit dem Computer. Ergebnisse von Beobachtungen und Befragungen im Bürobereich (S. 14-31). Bern, Göttingen, Toronto: Hans Huber.

Götz, R., Dahnncke, H. \& Langensiepen, F. (Hrsg.) (1992). Handbuch des Physikunterrichts. Sekundarbereich I. Band 5: Elektrizitätslehre I. Köln: Aulis Verlag Deubner \& Co KG.

Haeberlin, U. (1999). Reflexionen zur Bedeutung des heilpädagogischen Leitsatzes «Nicht gegen den Fehler, sondern für Fehlendes erziehen». In W. Althof (Hrsg), Fehlerwelten. Vom Fehlermachen und Lernen aus Fehlern (S. 89-99). Opladen: Leske + Budrich.

Hartinger, A. (1997). Aus Fehlern wird man klug - nur wie? Grundschule 29 (10), 29-30.

Heid, H. (1999). Autorität - Über die Verwandlung von Fehlern in Verfehlungen. In W. Althof (Hrsg.), Fehlerwelten. Vom Fehlermachen und Lernen aus Fehlern (S. 129-136). Opladen: Leske + Budrich.

Heuer, H. (1968). Die Englischstunde. Unterrichtsgestaltung und Unterrichtsforschung. Wuppertal, Ratingen: A. Henn Verlag.

Kahl, R. (1995). Lob des Fehlers. Hamburg: Pädagogische Beiträge Verlag.

Knapp-Potthoff, A. (1987). Fehler aus spracherwerblicher und sprachdidaktischer Sicht. Eine Bestandsaufnahme. Englisch Amerikanische Studien. Zeitschrift für Unterricht, Wissenschaft \& Politik 9 (2), 205-220.

Kobi, E. E. (1994). Fehler. Die neue Schulpraxis 64 (2), 5-10.

Loh, W. (1992). Dezision als Bestandteil einer Fortschrittsmoral. Gesamt-Kritik. Ethik und Sozialwissenschaften 3 (1), 68-74.

Lorenz, J. H. (2004). Mit Fehlern rechnen. Argumente für einen offenen Mathematikunterricht. Lernchancen 7 (39), 46-52.

Mach, E. (1980). Erkenntnis und Irrtum. Skizzen zur Psychologie der Forschung. Darmstadt: Wissenschaftliche Buchgesellschaft.

Oser, F., Hascher, T. \& Spychiger, M. (1999). Lernen aus Fehlern. Zur Psychologie des «negativen» Wissens. In W. Althof (Hrsg.), Fehlerwelten. Vom Fehlermachen und Lernen aus Fehlern (S. 11-41). Opladen: Leske + Budrich.

Reason, J. (1990). Human Error. Cambridge u. a.: Cambridge University Press.

Reusser, K. (1999). Schülerfehler - die Rückseite des Spiegels. In W. Althof (Hrsg). Fehlerwelten. Vom Fehlermachen und Lernen aus Fehlern (S. 203-231). Opladen: Leske + Budrich.

Ruf, U., Frei, N. \& Zimmermann, T. (2004). Wie Schüler aus ihren Fehlern lernen. Von der persönlichen Grammatik zur Klassengrammatik. In G. Becker u. a. (Hrsg.), Heterogenität. Unterschiede nutzen - Gemeinsamkeiten stärken. Friedrich Jahresheft XXII, 98-101.

Selz, O. (1922). Zur Psychologie des produktiven Denkens und des Irrtums. Eine experimentelle Untersuchung. Bonn: Friedrich Cohen.

Senders, J. W. \& Moray, N. P. (1991). Human Error: Cause, Prediction, and Reduction. Hillsdale (New Jersey) Hove, London: Lawrence Erlbaum Associates.

Spychiger, M., Oser, F., Hascher, T. \& Mahler, F. (1999). Entwicklung einer Fehlerkultur in der Schule. In W. Althof (Hrsg.), Fehlerwelten. Vom Fehlermachen und Lernen aus Fehlern (S. 43-70). Opladen: Leske + Budrich.

Weimer, H. (1929). Psychologie der Fehler. Leipzig: Julius Klinkhardt.

Weimer, H. (1939). Fehlerverhütung und Fehlervermeidung. Düsseldorf: Hub. Hoch.

Weinert, F. E. (1999). Aus Fehlern lernen und Fehler vermeiden lernen. In W. Althof (Hrsg.), 
Fehlerwelten. Vom Fehlermachen und Lernen aus Fehlern (S. 101-109). Opladen: Leske + Budrich.

Weingardt, M. (2004). Fehler zeichnen uns aus. Transdisziplinäre Grundlagen zur Theorie und Produktivität des Fehlers in Schule und Arbeitswelt. Bad Heilbrunn/Obb.: Klinkhardt.

Wertheimer, M. (1964). Produktives Denken. Frankfurt a. M.: Waldemar Kramer.

Wiater, W. (2004). Fehler = Helfer. Lernchancen 7 (39), 4-7.

Winter, H. (1995). Mathematikunterricht und Allgemeinbildung. Mitteilungen der Gesellschaft für Didaktik der Mathematik 61, 37-46.

Schlagworte: Didaktik, entdeckendes Lernen, Erwägung, Fehler, Grundschule, Irrtum, Kompetenz, Lehren, Misslingen, negatives Wissen, Reflexion, Scheitern, Schule, Unterricht

\section{Pour un nouveau traitement des erreurs durant les processus d'apprentissage et d'enseignement}

\section{Résumé}

L'article traite d'une description des concepts dans lesquels les auteurs présentent l'argument selon lequel durant les processus d'apprentissage et d'enseignement, les erreurs devraient être utilisées comme une aide au lieu de les appréhender avec angoisse. Par la suite, ils différencient les fautes possibles: les «bonnes» et les «mauvaises». Quelques exemples d'enseignement dans les écoles primaires, se présentant dans la vie quotidienne, montrent de toute évidence que le mot «erreur» è utilisé très hétérogènement. En considération de ce fait, il semble nécessaire de développer des projets profitant aux élèves ainsi qu'aux enseignants. Par cela, ceux-ci deviennent plus sensibles à la fonction positive de l'erreur. Finalement, l'article présente comme argument qu'une erreur pourrait être envisagée positivement, et de plus, pourrait lui être attribuée des nouvelles alternatives.

Mots clés: apprendre, bévue, défaut, délibération, didactique, école primaire, enseigner, erreur, faute, méprise

\section{Per un trattamento innovativo degli «errori» nei processi d'apprendimento e d'insegnamento}

\section{Riassunto}

L'articolo si apre con una descrizione dei concetti riguardando il trattamento degli errori. In questo discorso, gli autori discutono su come possono essere migliorati i processi d'apprendimento e d'insegnamento senza incutere timore. Per questo è importante distinguere gli errori «costruttivi» da quelli «dannosi». Prendendo spunto da alcuni esempi della vita quotidiana nelle scuole elementari, è stato dimostrato che gli «errori» sono eterogeni. Tenendo presente questo, è ne- 
cessario sviluppare dei concetti che aiutanto gli alunni e gli insegnanti ad essere più riflessivi. Solo in questo modo possono diventare più sensibili agli errori. Alla fine, è stata dimostrata la funzione positiva degli errori che, se sopportati da giustificazione, possono essere costruttivi.

Parole chiave: abbaglio, abilità, competenza, deliberazione, didattica, difetto, errore, imparare, insegnamento, insegnare, ponderazione, sbaglio, scuola

\section{Active looking for mistakes in learning and teaching processes and deliberative orientation}

\section{Summary}

The article starts with a description of concepts whose authors argue that mistakes should be used as constructive means in learning and teaching processes instead of anxiously avoiding them. Nevertheless, one has to differentiate between "good» and "bad mistakes». A closer look at some examples of mistakes as they happen in everyday life of primary schools shows that the word "mistake» is used very heterogeneously. Taking this into account it seems necessary to develop concepts which help learners and teachers to become more reflective in saying what might be a mistake or something else. Furthermore it is argued that the positive function of mistakes could be supported if deliberated alternatives were appreciated in the context of justification.

Keywords: ability, competence, deliberation, didactic, error, learning, mistake, negative knowledge, primary school, slip, teaching 